\title{
Diagnóstico y tratamiento de pacientes con telangiectasia hemorrágica hereditaria (síndrome de Rendu-Osler-Weber) en un hospital universitario en Colombia
}

\section{Diagnosis and treatment of patients with hereditary hemorrhagic telangiectasia (Rendu-Osler-Weber syndrome) at a university hospital in Colombia}

\author{
Gabriel Alonso Mosquera-Klinger, ${ }^{1 *}$ Kenny Gálvez Cárdenas, ${ }^{2}$ Ana María Valencia. ${ }^{3}$
}

\footnotetext{
1 Especialista en Medicina Interna, Gastroenterología y Endoscopia Digestiva, Hospital Pablo Tobón Uribe. Medellín, Colombia.

2 Medicina Interna y Hematología, Hospital Pablo Tobón Uribe. Medellín, Colombia.

3 Especialista en Medicina Interna, Universidad Pontificia Bolivariana. Medellín, Colombia.

*Correspondencia: gami8203@yahoo.com.
}

\begin{abstract}
Resumen
Introducción: la telangiectasia hemorrágica hereditaria $(\mathrm{THH})$ es una enfermedad vascular hereditaria caracterizada por epistaxis, sangrado digestivo y anemia crónica; en muchos casos hay malformaciones arteriovenosas de órganos sólidos. El diagnóstico se realiza con base en datos clínicos, hallazgos endoscópicos e imagenológicos. La detección temprana con enfoque multidisciplinario y tratamiento de las complicaciones impacta en morbimortalidad de la enfermedad. Objetivos: describir las características demográficas, clínicas y desenlaces de pacientes con diagnóstico de THH en un hospital universitario. Métodos: estudio tipo serie de casos en pacientes evaluados entre 2012 hasta el 2017. Resultados: se obtuvieron registros de 18 casos, $11(61,1 \%)$ hombres, con edad mediana de 56 años (rango intercuartílico [IQR]: 52-64). Los casos son provenientes de Colombia y algunos países caribeños. En todos los pacientes el diagnóstico se estableció mediante los criterios de Curazao. El número de ingresos hospitalarios tuvo una mediana de 6 días (IQR: 2,520,5). Los ingresos fueron en relación a sangrado en todos los casos, $61 \%$ de los pacientes requirió transfusión de hemoderivados. En el $61 \%$ de los pacientes se identificó compromiso en el órgano sólido mediante imágenes. Conclusiones: la THH es una enfermedad de expresión clínica variable. En nuestro estudio las manifestaciones gastrointestinales fueron las causas de ingreso más frecuentes. Se requirió con frecuencia transfusión de hemoderivados. Los pacientes requirieron múltiples estudios para identificar la extensión de la enfermedad y el compromiso de órgano sólido. El tratamiento se basó en el manejo endoscópico y médico, especialmente a base de bevacizumab y octreotida.
\end{abstract}

Palabras clave

Telangiectasia hemorrágica hereditaria, hemorragia, epistaxis, melena.

\begin{abstract}
Introduction: Hereditary hemorrhagic telangiectasia (HHT or Osler-Weber-Rendu syndrome) is a hereditary vascular disease characterized by recurrent epistaxis, gastrointestinal bleeding and chronic anemia. Many cases have arteriovenous malformations of solid organs. Diagnosis is based on clinical data, endoscopy and imaging. Early detection and treatment of complications with a multidisciplinary approach impacts the disease's morbidity and mortality. Objectives: The objective of this study was to describe the demographic, clinical and outcome characteristics of patients diagnosed with HHT at a university hospital. Methods: This is a case series of patients evaluated between 2012 and 2017. Results: Records of 18 cases were obtained. The patients were from Colombia and other Caribbean countries. All diagnoses were established using the Curaçao criteria. Eleven patients 11 (61.1\%) were men, and the median patient age was 56 years (IQR 52-64). The median number of hospital admissions was 6 (33.3\%) (IQR 2.5-20.5), and all admissions were related to bleeding. Sixty-one percent of patients required transfusion of blood products, and the compromises of solid organs were found in the same number of patients by imaging studies. Conclusions: The clinical expression of THH varies, but in our study gastrointestinal manifestations were the most frequent causes of hospital admission. They frequently required transfusion of blood products and patients required multiple studies to identify the extent of the disease, and solid organ compromise. Treatment was based on endoscopic and medical management, especially administration of bevacizumab and octreotide.
\end{abstract}

\section{Keywords}

Hereditary hemorrhagic telangiectasia, hemorrhage, epistaxis, melena. 


\section{INTRODUCCIÓN}

La telangiectasia hemorrágica hereditaria $(\mathrm{THH})$ o síndrome de Rendu-Osler-Weber (SOWR) es una enfermedad vascular hereditaria autosómica dominante con diferentes manifestaciones clínicas. Los pacientes suelen presentar epistaxis, hemorragia gastrointestinal y anemia por deficiencia de hierro debido a telangiectasias mucocutáneas. Se ha descrito que los pacientes con THH también corren el riesgo de desarrollar malformaciones arteriovenosas especialmente en la circulación cerebral, pulmonar y hepática, que pueden causar un grave daño orgánico (1). La detección inicial del SOWR se basa en datos clínicos y a menudo se utilizan los criterios de Curazao (Tabla 1), que consisten en epistaxis recurrente, telangiectasias, malformaciones vasculares viscerales y un familiar de primer grado con SOWR (el diagnóstico establecido se realiza con 3 o más de estos criterios) (2,3). El tratamiento de esta entidad consiste en el manejo de los síntomas y de las complicaciones (3).

Tabla 1. Criterios diagnósticos de Curazao para telangiectasia hemorrágica hereditaria

\begin{tabular}{|c|c|}
\hline \multirow[t]{3}{*}{ Criterios } & $\begin{array}{l}\text { Epistaxis o hemorragia nasal espontánea y recurrente. } \\
\text { Telangiectasias en múltiples sitios característicos } \\
\text { (labios, cavidad oral, dedos y nariz). }\end{array}$ \\
\hline & $\begin{array}{l}\text { Lesiones viscerales, telangiectasias en el tracto } \\
\text { gastrointestinal (con o sin sangrado) o malformaciones } \\
\text { arteriovenosas pulmonares, hepáticas, cerebrales y } \\
\text { espinales. }\end{array}$ \\
\hline & $\begin{array}{l}\text { Antecedentes familiares: familiar en primer grado con } \\
\text { diagnóstico de THH. }\end{array}$ \\
\hline \multirow{3}{*}{$\begin{array}{l}\text { Clasificación } \\
\text { de la } \\
\text { enfermedad }\end{array}$} & Definitiva: si se cumplen 3 o más criterios. \\
\hline & Posible o presunta: si se cumplen 2 criterios. \\
\hline & Poco probable: si se cumplen menos de 2 criterios. \\
\hline
\end{tabular}

La prevalencia estimada de la THH es 1,5-2 personas por $10000(4,5)$. Algunos autores describen que la penetrancia variable (completa e incompleta) podría impactar en el reconocimiento de la enfermedad y en la notificación de la misma, ya que no todos los pacientes presentan síntomas en edades tempranas $(3,4)$. Esta entidad tiene una mayor prevalencia en ciertas poblaciones, como en afrocaribeños de Curazao y Bonaire (5). Hasta el momento hay escasas series de casos publicadas en América Latina, pero sí hay reportes de casos aislados en los que se describen manifestaciones clínicas variables y también el compromiso en órganos sólidos por malformaciones arteriovenosas (6-8).

El objetivo de este trabajo es describir las características demográficas y clínicas y los desenlaces de pacientes con diagnóstico de $\mathrm{THH}$ en un hospital universitario.

\section{MATERIALES Y MÉTODOS}

Es un estudio tipo serie de casos. Se realizó en pacientes mayores de 18 años, con diagnóstico establecido de THH o SOWR en el hospital, en el período de enero de 2012 a julio de 2017. El protocolo fue aprobado por el comité de ética e investigaciones del Hospital Pablo Tobón Uribe, Medellín, Colombia. No se requirió autorización escrita, ya que en los datos publicados no se revelaron nombres, datos personales de identidad, ni fotos que permitan reconocer al individuo. De acuerdo con la Resolución Nacional 8430 de 1993, el estudio se clasifica como de riesgo mínimo, es decir, que no pone en riesgo la integridad ni la identidad del paciente.

Se realizó la revisión de las historias clínicas y de los procedimientos realizados en los individuos a estudio en el período comprendido entre enero de 2012 y julio de 2017.

\section{Forma de recolección y análisis de datos}

La recolección de las variables fue realizada por los investigadores en una base de datos. Se utilizó estadística descriptiva mediante medidas de tendencia central como el promedio, mediana y rangos.

\section{RESULTADOS}

Se obtuvieron registros de 18 casos (Tablas 2 y 3 ): 11 hombres $(61,1 \%)$ y 9 mujeres $(29,9 \%)$, con mediana de edad de 56 años (rango intercuartílico [IQR]: 52-64); 6 provenientes de Antioquia (33,3\%), 4 de Chocó (22,2 \%), 2 de Caldas (11,2\%), 1 de Quindío (5,5\%), 1 de Valle (5,5\%), 3 de Bonaire (16,7 \%) y 1 de Curazao (5,5\%).

El 55,55 \% refirió historia familiar de primer grado de THH, 6 casos $(33,33 \%)$ fueron espontáneos y en solo 2 personas $(11,11 \%)$ no había datos referentes en los antecedentes en la historia clínica. Los 2 pacientes de Riosucio, Caldas, 1 de Curazao y 1 de Bonaire tenían el antecedente familiar de primer grado; así mismo, 3/4 (75\%) de los provenientes del Chocó hicieron referencia de su historia familiar de THH.

Los síntomas referidos al momento del ingreso fueron: epistaxis en el 66,7 \%, melenas en el $50 \%$, hematoquecia en el 22,22 \%, astenia-adinamia en el $27,8 \%$, fatiga en el $11,11 \%$, disnea en el 11,11 \% y otros síntomas como hemoptisis fue referido por 1 paciente; equimosis y hemorragia uterina anormal (HUA) por otro paciente.

Se realizaron estudios endoscópicos en $88,8 \%$ de los pacientes, en el resto no se registraron estudios en la historia clínica. A todos los pacientes con estudios endoscópicos se les registró endoscopia digestiva superior, videocápsula endoscópica (VCE) en 13/16 (81,25\%), enteroscopia en el $50 \%$ y colonoscopia también en el $50 \%$. 
Tabla 2. Características demográficas y clínicas de pacientes con THH

\begin{tabular}{|c|c|c|c|c|c|c|c|}
\hline Caso & $\begin{array}{l}\text { Edad/ } \\
\text { sexo }\end{array}$ & Procedencia & $\begin{array}{l}\text { Historia } \\
\text { familiar }\end{array}$ & Síntomas de consulta & $\begin{array}{c}\text { Conteo } \\
\text { mínimo de } \\
\text { plaquetas }\end{array}$ & $\begin{array}{l}\text { Nivel mínimo de } \\
\text { hemoglobina }\end{array}$ & $\begin{array}{l}\text { Compromiso de } \\
\text { órgano sólido }\end{array}$ \\
\hline 1 & $58 / \mathrm{M}$ & Riosucio, Caldas & Sí & $\begin{array}{l}\text { Astenia, adinamia, fatiga, disnea, } \\
\text { epistaxis, melena, hematoquecia }\end{array}$ & 88000 & $3,7 \mathrm{~g} / \mathrm{dL}$ & Sí (hígado) \\
\hline 2 & $62 / \mathrm{F}$ & Cali & ND & $\begin{array}{l}\text { Hematoquecia, astenia, adinamia, } \\
\text { disnea }\end{array}$ & 144000 & $6,3 \mathrm{~g} / \mathrm{dL}$ & $\begin{array}{l}\text { Sí (hígado, pulmón, } \\
\text { páncreas) }\end{array}$ \\
\hline 3 & $52 / M$ & Curazao & Sí & Epistaxis, sangrado gastrointestinal & 125000 & $5,4 \mathrm{~g} / \mathrm{dL}$ & Sí (hígado) \\
\hline 4 & $41 / F$ & Medellín, Antioquia & No & HUA, equimosis espontáneas & 359000 & $12,6 \mathrm{~g} / \mathrm{dL}$ & Ninguno \\
\hline 5 & $77 / M$ & Andes, Antioquia & No & Melena & 166000 & $5,1 \mathrm{~g} / \mathrm{dL}$ & Ninguno \\
\hline 6 & $54 / \mathrm{F}$ & Riosucio, Caldas & Sí & Epistaxis, melena, hematoquecia & 208000 & $7,4 \mathrm{~g} / \mathrm{dL}$ & Ninguno \\
\hline 7 & $63 / M$ & Curazao & Sí & Hemoptisis, melena, epistaxis & 231000 & $5,3 \mathrm{~g} / \mathrm{dL}$ & Sí (pulmón) \\
\hline 8 & $59 / F$ & Lloró, Chocó & Sí & Epistaxis, melenas, hematoquecia & ND & $8,7 \mathrm{~g} / \mathrm{dL}$ & Ninguno \\
\hline 9 & $56 / F$ & Lloró, Chocó & No & Astenia, adinamia, epistaxis & 131700 & $7,6 \mathrm{~g} / \mathrm{dL}$ & $\begin{array}{l}\text { Sí (hígado, pulmón, } \\
\text { cerebro) }\end{array}$ \\
\hline 10 & $78 / \mathrm{M}$ & Bonaire & ND & $\begin{array}{l}\text { Epistaxis, astenia, adinamia, fatiga, } \\
\text { palidez }\end{array}$ & 224000 & $7,4 \mathrm{~g} / \mathrm{dL}$ & Sí (pulmón) \\
\hline 11 & $67 / M$ & La Unión, Antioquia & No & Melena & 110000 & $3,6 \mathrm{~g} / \mathrm{dL}$ & Sí (pulmón) \\
\hline 12 & $58 / \mathrm{M}$ & Bonaire & No & Melena & 416000 & $8,9 \mathrm{~g} / \mathrm{dL}$ & Ninguno \\
\hline 13 & $52 / M$ & Bello, Antioquia & Sí & Epistaxis & 241000 & $16 \mathrm{~g} / \mathrm{dL}$ & Sí (hígado) \\
\hline 14 & $56 / F$ & Quibdó, Chocó & Sí & Melena, epistaxis & 134000 & $4,9 \mathrm{~g} / \mathrm{dL}$ & Ninguno \\
\hline 15 & $27 / M$ & Lloró, Chocó & Sí & Epistaxis & ND & ND & Sí (pulmón, cerebro) \\
\hline 16 & $72 / M$ & Riosucio, Caldas & Sí & Astenia, adinamia & 262000 & $7,1 \mathrm{~g} / \mathrm{dL}$ & Ninguno \\
\hline 17 & $48 / \mathrm{F}$ & Medellín, Antioquia & No & Epistaxis & 264000 & ND & Sí, (pulmón, cerebro) \\
\hline 18 & $55 / M$ & Medellín, Antioquia & Sí & Epistaxis, dolor abdominal & 234000 & $6 \mathrm{~g} / \mathrm{dL}$ & Sí (hígado) \\
\hline
\end{tabular}

F: femenino; HUA: hemorragia uterina anormal; M: masculino; ND: no hay datos.

El 55,55 \% de los pacientes ingresó al hospital por urgencias en relación con el sangrado digestivo manifestado por melena o hematoquecia, la hemoglobina mínima de ingreso en este grupo de pacientes estuvo en rangos de 3,6-8,9 g/dL. De estos, se les realizaron estudios endoscópicos a todos. Al $100 \%$ de estos casos se les realizó VCE (Tabla 3), de los cuales el $70 \%$ requirió enteroscopia con fines terapéuticos (aplicación de argón plasma sobre lesiones con potencial riesgo de sangrado o sangrantes) (Figura 1).

En cuanto a la terapia médica prescrita, el bevacizumab se administró en el 27,7\% de los casos, octreotida en el 11,1\%, talidomida en el 5,55 \% y tamoxifeno en el 5,55 \%.

El número de ingresos hospitalarios tuvo una mediana de 6 días (IQR: 2,5-20,5), todos los casos estuvieron relacionados con sangrado digestivo; el $61 \%$ de los pacientes requirió transfusión de hemoderivados (Tabla 3). En el $61 \%$ de los pacientes se identificó compromiso en órgano sólido por malformaciones arteriovenosas (hígado, pulmón, cerebro o páncreas): la afectación exclusiva del hígado se presentó en 4 de ellos (22,2\%); el compromiso exclusivo del pulmón en 3 individuos (16,7\%); en el resto de pacientes el compromiso fue multiorgánico: 1 paciente en el hígado, pulmón y páncreas; en otro paciente, hígado, pulmón y cerebro; y en otros 2 pacientes, pulmón y cerebro.

En la Tabla 2 se describe el sexo, edad, exámenes hematológicos tomados durante la hospitalización (conteo mínimo de plaquetas y de la hemoglobina) y el compromiso de órgano sólido por malformación arteriovenosa. En la Tabla 3 se anotan las manifestaciones clínicas y los hallazgos físicos visibles, además de la distribución de las telangiectasias en el tracto digestivo (Figura 2); también se describen las terapias recibidas y las transfusiones.

\section{DISCUSIÓN}

En nuestra serie, la mayoría de los pacientes tuvo episodios menores de sangrado que fueron subestimados en edades tempranas. En varias revisiones se describe que más de la mitad de los pacientes presenta síntomas antes de los 20 años de edad y la prevalencia de la epistaxis puede ser 
Tabla 3. Datos clínicos, tratamientos recibidos de los casos con $\mathrm{THH}$

\begin{tabular}{|c|c|c|c|c|c|c|}
\hline Casos & $\begin{array}{c}\text { Ingresos } \\
\text { hospitalarios }\end{array}$ & $\begin{array}{c}\text { Estigmas } \\
\text { periféricos: } \\
\text { mucocutáneos }\end{array}$ & $\begin{array}{c}\text { Telangiectasias en el tracto } \\
\text { digestivo }\end{array}$ & $\begin{array}{l}\text { Terapia médica } \\
\text { recibida }\end{array}$ & $\begin{array}{l}\text { Procedimientos } \\
\text { endoscópicos } \\
\text { realizados }\end{array}$ & $\begin{array}{c}\text { Transfusión de } \\
\text { hemoderivados } \\
\text { (unidades) }\end{array}$ \\
\hline 1 & 74 & Nariz, manos & $\begin{array}{l}\text { Esófago, estómago, duodeno, } \\
\text { yeyuno, íleon, colon }\end{array}$ & $\begin{array}{l}\text { Endoscópica, } \\
\text { bevacizumab }\end{array}$ & $\begin{array}{l}\text { EDS 8, enteroscopia 1, } \\
\text { VCE } 1\end{array}$ & Sí (179 U) \\
\hline 2 & 40 & ND & Esófago, estómago, duodeno & $\begin{array}{l}\text { Endoscópica, } \\
\text { bevacizumab }\end{array}$ & EDS 10, VCE & Sí (2 U) \\
\hline 3 & 15 & Lengua & Duodeno, yeyuno & $\begin{array}{l}\text { Endoscópica, } \\
\text { bevacizumab, } \\
\text { talidomida, tamoxifeno }\end{array}$ & $\begin{array}{l}\text { EDS 2, VCE 1, } \\
\text { enteroscopia } 1\end{array}$ & No \\
\hline 4 & 11 & Manos y tronco & ND & Ninguno & ND & No \\
\hline 5 & 9 & Cara & $\begin{array}{l}\text { Esófago, estómago, duodeno, } \\
\text { yeyuno }\end{array}$ & Endoscópica & $\begin{array}{l}\text { EDS } 6 \text {, colonoscopia } 1, \\
\text { VCE } 1 \text {, enteroscopia } 1\end{array}$ & Sí (35 U) \\
\hline 6 & 37 & $\begin{array}{l}\text { Lengua, dedos } \\
\text { de las manos }\end{array}$ & Estómago, colon & Endoscópica & $\begin{array}{c}\text { EDS 19, colonoscopia } \\
\text { 2, VCE } 1\end{array}$ & Sí (1 U) \\
\hline 7 & 46 & $\begin{array}{l}\text { Lengua, labios, } \\
\text { Manos }\end{array}$ & $\begin{array}{l}\text { Esófago, estómago, duodeno, } \\
\text { yeyuno }\end{array}$ & $\begin{array}{l}\text { Endoscópica, } \\
\text { bevacizumab }\end{array}$ & $\begin{array}{l}\text { EDS 5, colonoscopia 1, } \\
\text { enteroscopia 1, VCE } 1\end{array}$ & Sí (6 U) \\
\hline 8 & 7 & Lengua, paladar & $\begin{array}{l}\text { Hipofaringe, estómago, } \\
\text { duodeno, yeyuno }\end{array}$ & Endoscópica & $\begin{array}{l}\text { EDS 2, colonoscopia } \\
\text { 1, VCE } 1\end{array}$ & ND \\
\hline 9 & 8 & ND & Estómago, duodeno & Endoscópica & $\begin{array}{l}\text { EDS 1, colonoscopia } \\
1, \text { VCE } 1\end{array}$ & Si $(2 \mathrm{U})$ \\
\hline 10 & 5 & ND & Estómago, duodeno & Endoscópica & $\begin{array}{l}\text { EDS 2, VCE 1, } \\
\text { enteroscopia } 1\end{array}$ & Sí (1 U) \\
\hline 11 & 3 & $\begin{array}{l}\text { Labios, paladar, } \\
\text { nariz }\end{array}$ & Estómago & Endoscópica & $\begin{array}{c}\text { EDS 19, colonoscopia } \\
\text { 2, VCE } 1\end{array}$ & Sí (13U) \\
\hline 12 & 4 & ND & $\begin{array}{l}\text { Estómago, duodeno, yeyuno, } \\
\text { íleon }\end{array}$ & Endoscópica, octreotida & $\begin{array}{l}\text { EDS 3, VCE 1, } \\
\text { enteroscopia } 1\end{array}$ & No \\
\hline 13 & 3 & Lengua & Ninguna & Ninguna & EDS 1 & No \\
\hline 14 & 3 & Lengua, labios & Estómago, duodeno, yeyuno & Endoscópica & $\begin{array}{l}\text { EDS 8, colonoscopia } 1, \\
\text { VCE 1, enteroscopia } 1\end{array}$ & Sí (32 U) \\
\hline 15 & 1 & ND & ND & Bevacizumab & ND & ND \\
\hline 16 & 1 & $\begin{array}{l}\text { Nariz, lengua, } \\
\text { labios }\end{array}$ & Estómago, duodeno, yeyuno & Endoscópica, octreotida & $\begin{array}{l}\text { EDS 1, colonoscopia 1, } \\
\text { enteroscopia 1, VCE } 1\end{array}$ & Si (2 U) \\
\hline 17 & 1 & ND & Estómago & Ninguna & EDS 1 & ND \\
\hline 18 & 1 & Lengua, labios & Estómago, duodeno, yeyuno & Endoscópica & EDS 1 & Sí (2 U) \\
\hline
\end{tabular}

EDS: endoscopia digestiva superior; U: unidades; VCE: videocápsula endoscópica.

incluso mayor del $90 \%$ de los casos $(9,10)$. Los síntomas descritos con más frecuencia fueron epistaxis, seguidos por melena, hematoquecia y síntomas generales de pérdidas hemáticas como astenia, adinamia, fatiga e incluso disnea. La mayoría tenía anemia grave, con síntomas variados en relación con esta y con necesidad de transfusión de hemoderivados, como se describió en una revisión sistemática reciente (3). El número de unidades de glóbulos rojos transfundidas tuvo una clara relación con la extensión y gravedad de la enfermedad.

En nuestra serie, a ningún paciente se le realizaron estudios genéticos para determinar la presencia de genes rela- cionados con la THH. Estos exámenes no están ampliamente disponibles en nuestro medio y su realización es costosa; además, existe controversia sobre la expresión clínica variable. En un estudio reciente no se encontraron diferencias significativas en la mortalidad en un período mayor de 90 meses entre THH tipos 1 y 2 (11).

En los pacientes a quienes se les documentó telangiectasias en el tracto digestivo, las lesiones se encontraron mayormente en una localización proximal. El estómago, duodeno y yeyuno fueron los sitios más comunes. En la mayoría de casos se utilizó la VCE como método no invasivo para evaluar el compromiso en el intestino delgado y 

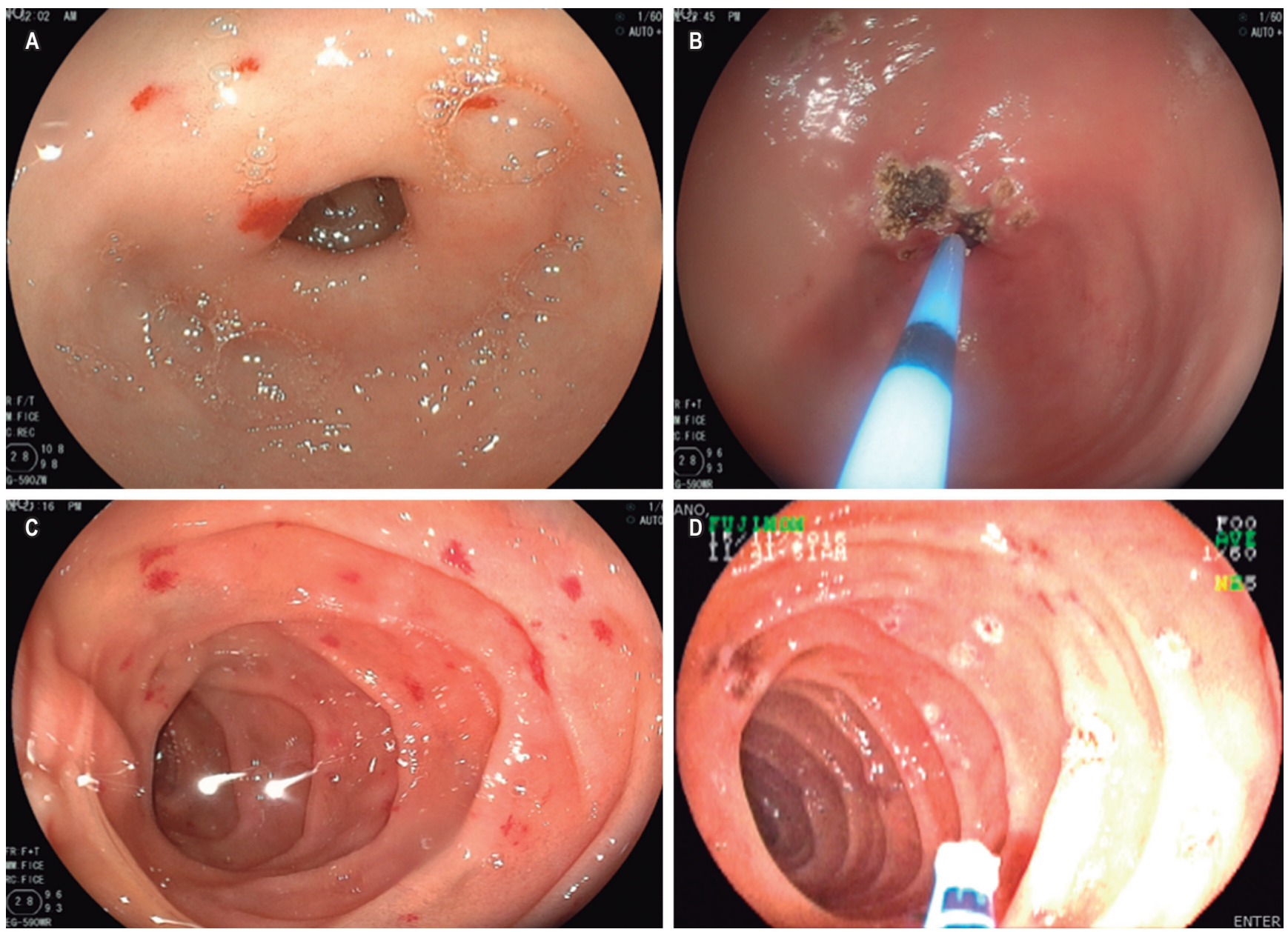

Figura 1. A. Telangiectasias en el antro. B. Terapia con argón plasma sobre las lesiones en el antro. C. Telangiectasias en el intestino delgado. D. Terapia con argón plasma.
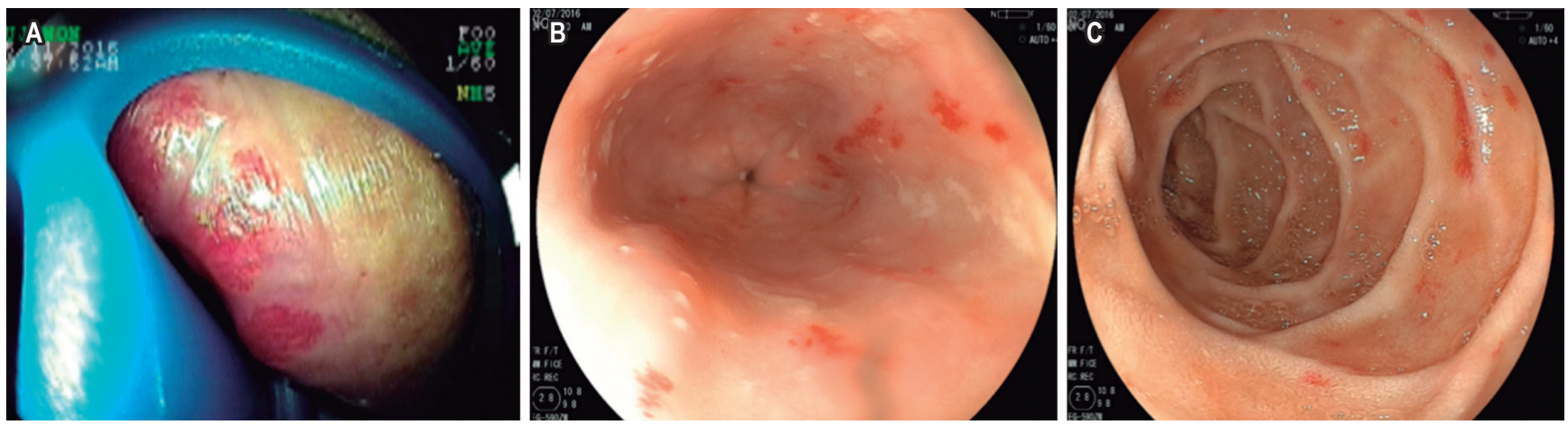

Figura 2. Compromiso extenso por THH. A. Labios. B. Múltiples telangiectasias en el estómago. C. Telangiectasias en el duodeno.

definir la necesidad de terapia endoscópica. Estos datos son similares a lo descrito en la revisión sistemática de Jackson y colaboradores (3). En los casos en los que se evidenció sangrado en el intestino delgado, se utilizó enteroscopia de doble balón con fines terapéuticos con aplicación de terapia argón plasma, con o sin terapia sistémica. 
La terapia sistémica se utilizó en casos con sangrado refractario. Teniendo en cuenta que se ha descrito que los pacientes con esta entidad tienen aumento en la producción del factor de crecimiento del endotelio vascular, también podría haber un desbalance entre factores antiangiogénicos y proangiogénicos $(12,13)$. Esto ha permitido utilizar medicamentos cuyo mecanismo de acción sea inhibir el factor de crecimiento del endotelio vascular como el bevacizumab. Hay múltiples reportes de casos en adultos como el descrito por Combariza y colaboradores (14) del Hospital Pablo Tobón Uribe en Medellín. También se ha usado en pequeñas series de casos en las que se resaltan buenos resultados en cuanto a la efectividad y seguridad $(15,16)$. Los costos y el perfil de eventos adversos del medicamento no son despreciables (17), por lo que se debe hacer una adecuada elección del paciente para definir dicha terapia. No obstante, creemos que podría ser un medicamento prometedor en el escenario de compromiso multiorgánico y sangrado refractario.

El compromiso por malformaciones arteriovenosas en órgano sólido se identificó en más del $60 \%$ de los casos, estos en su mayoría tenían compromiso pulmonar o hepático, seguidos de afectación cerebral. En este aspecto, lo descrito en la literatura es variable en cuanto a métodos utilizados para detectar dichas malformaciones. Jackson y colaboradores encontraron que los expertos temáticos recomiendan estudiar las malformaciones arteriovenosas pulmonares con ecocardiograma transtorácico contrastado y, en caso de que se encuentren hallazgos anormales, complementan con tomografía axial computarizada (TAC) torácica de alta resolución (3). Las malformaciones vasculares hepáticas se estudian en pacientes con $\mathrm{THH}$ confirmado cuando tengan alteración en las pruebas de función hepática, colestasis, hipertensión portal o insuficiencia cardíaca derecha. El estudio de estos casos se realiza mediante ecografía hepática con Doppler o TAC helicoidal trifásico (18-20). En nuestra serie, a todos los casos se les estudió mediante resonancia magnética nuclear (RMN) contrastada de abdomen; este es un examen disponible en nuestro hospital, con alta experiencia y tiene menos riesgo de nefrotoxicidad en comparación con el contraste yodado, por lo que se considera de elección en este escenario.

Se identificaron alteraciones arteriovenosas cerebrales en el $16,6 \%$, dato muy parecido a lo descrito por Fulbright y colaboradores, quienes mencionaron una prevalencia del 10 $\%$ con resonancia cerebral (21). La resonancia cerebral es el método más utilizado después de los 18 años en pacientes asintomáticos con $\mathrm{THH}$ posible o confirmada (3).

En cuanto al pronóstico, la mayoría de individuos con THH que tienen un acceso bueno a los servicios de salud tiene una expectativa de vida normal en relación con la población general (3). Hay una distribución bimodal de la mortalidad, con picos a los 50 años y entre los 60 y 79 años. Las complicaciones agudas en relación con las malformaciones arteriovenosas son la causa principal de la muerte, en especial en el contexto de un inadecuado cuidado de la salud, ya que juega un papel fundamental el seguimiento de estos pacientes $(3,22)$.

Consideramos que se requieren más estudios poblacionales para determinar la prevalencia local real, además de estudios prospectivos en los cuales se planteen alternativas de tratamiento que impacten en menos tasas de morbilidad y menos hospitalizaciones; y también propuestas para el seguimiento de familiares de primer grado asintomáticos.

\section{REFERENCIAS}

1. Sharathkumar AA, Shapiro A. Hereditary haemorrhagic telangiectasia. Haemophilia. 2008;14(6):1269-80. https:// doi.org/10.1111/j.1365-2516.2008.01774.x.

2. GuttmacherAE,MarchukDA, White RIJr. Hereditaryhemorrhagic telangiectasia. N Engl J Med. 1995;333(14):918-24. https://doi.org/10.1056/NEJM199510053331407.

3. Jackson SB, Villano NP, Benhammou JN, Lewis M, Pisegna JR, Padua D. Gastrointestinal Manifestations of Hereditary Hemorrhagic Telangiectasia (HHT): A Systematic Review of the Literature. Dig Dis Sci. 2017;62(10):2623-30. https://doi.org/10.1007/s10620-017-4719-3.

4. Dakeishi M, Shioya T, Wada Y, Shindo T, Otaka K, Manabe $\mathrm{M}$, et al. Genetic epidemiology of hereditary hemorrhagic telangiectasia in a local community in the northern part of Japan. Hum Mutat. 2002;19(2):140-8. https://doi. org/10.1002/humu.10026.

5. Grosse SD, Boulet SL, Grant AM, Hulihan MM, Faughnan ME. The use of US health insurance data for surveillance of rare disorders: hereditary hemorrhagic telangiectasia. Genet Med. 2014;16(1):33-9. https://doi.org/10.1038/gim.2013.66.

6. GómezMA, Ruiz O, Otero W. Telangiectasia hemorrágica hereditaria. Reporte de caso. Rev Col Gastrenterol 2015;30(4):46973. https://doi.org/10.22516/25007440.11.

7. Sandoval DK, García E, Ramírez S, Torres KJ, Velandia MC, Villamizar JF, et al. Síndrome de Rendu Osler Weber en una adolescente en Colombia. Reporte de un caso de autopsia. Biosalud. 2018;17(1):83-9.

8. Giraldo A, Conde R, Varón F. Hipertensión pulmonar como manifestación de la telangiectasia hemorrágica hereditaria o síndrome de Osler-Weber-Rendú. Rev Col Neumol. 2014;26(3):139-144. https://doi.org/10.30789/rcneumologia.v26.n3.2014.39.

9. Alcalá-Villalón T, Castillo-González D, Agramonte-Llanes O. Enfermedad de Rendú-Osler-Weber: A propósito de 5 casos con epistaxis recurrente. Rev Cubana Hematol Inmunol Hemoter. 2012;28(3):289-98.

10. Faughnan ME, Palda VA, Garcia-Tsao G, Geisthoff UW, McDonald J, Proctor DD, et al. International guidelines for the diagnosis and management of hereditary haemorrhagic 
telangiectasia. J Med Genet. 2011;48(2):73-87. https://doi. org/10.1136/jmg.2009.069013.

11. Kjeldsen AD, Møller TR, Brusgaard K, Vase P, Andersen PE. Clinical symptoms according to genotype amongst patients with hereditary haemorrhagic telangiectasia. J Intern Med. 2005;258(4):349-55. https://doi.org/10.1111/j.13652796.2005.01555.x.

12. Heldin $\mathrm{CH}$, Miyazono $\mathrm{K}$, ten Dijke P. TGF-beta signalling from cell membrane to nucleus through SMAD proteins. Nature. 1997 Dec 4;390(6659):465-71. https://doi. org/10.1038/37284.

13. Sadick H, Riedel F, Naim R, Goessler U, Hörmann K, Hafner $\mathrm{M}$, et al. Patients with hereditary hemorrhagic telangiectasia have increased plasma levels of vascular endothelial growth factor and transforming growth factor-betal as well as high ALK1 tissue expression. Haematologica. 2005;90(6):818-28.

14. Combariza JF, Olaya VP. Telangiectasia hemorrágica hereditaria. Síndrome de Osler Weber Rendú y manejo con bevacizumab. Acta Med Colomb. 2015;40:66-8.

15. BoseP,HolterJL, Selby GB.Bevacizumabinhereditaryhemorrhagic telangiectasia. N Engl J Med. 2009;360(20):2143-4. https://doi.org/10.1056/NEJMc0901421.

16. Dupuis-Girod S, Ginon I, Saurin JC, Marion D, Guillot E, Decullier E, et al. Bevacizumab in patients with hereditary hemorrhagic telangiectasia and severe hepatic vascular malformations and high cardiac output. JAMA. 2012;307(9):94855. https://doi.org/10.1001/jama.2012.250.
17. Garg N, Khunger M, Gupta A, Kumar N. Optimal management of hereditary hemorrhagic telangiectasia. J Blood Med. 2014;5:191-206. https://doi.org/10.2147/JBM.S45295.

18. Ravard G, Soyer P, Boudiaf M, Terem C, Abitbol M, Yeh JF, et al. Hepatic involvement in hereditary hemorrhagic telangiectasia: helical computed tomography features in 24 consecutive patients. J Comput Assist Tomogr. 2004;28(4):488-95.

19. Barral M, Sirol M, Placé V, Hamzi L, Borsik M, Gayat E, et al. Hepatic and pancreatic involvement in hereditary hemorrhagic telangiectasia: quantitative and qualitative evaluation with 64-section CT in asymptomatic adult patients. Eur Radiol. 2012;22(1):161-70. https://doi.org/10.1007/s00330-0112243-y.

20. Garcia-Tsao G, KorzenikJR, Young L, Henderson KJ, Jain D, Byrd B, et al. Liver disease in patients with hereditary hemorrhagic telangiectasia. N Engl J Med. 2000;343(13):9316./10.1056/NEJM200009283431305.

21. Fulbright RK, Chaloupka JC, Putman CM, Sze GK, Merriam MM, Lee GK, et al. MR of hereditary hemorrhagic telangiectasia: prevalence and spectrum of cerebrovascular malformations. AJNR Am J Neuroradiol. 1998;19(3):477-84.

22. Sabbà C, Pasculli G, Suppressa P, D'Ovidio F, Lenato GM, Resta F, et al. Life expectancy in patients with hereditary haemorrhagic telangiectasia. QJM. 2006;99(5):327-34. https://doi.org/10.1093/qjmed/hcl037. 